\title{
High-throughput label-free characterization of viable, necrotic and apoptotic human lymphoma cells in a coplanar-electrode microfluidic impedance chip
}

\author{
Adele De Ninno $^{\mathrm{a}}$, Riccardo Reale ${ }^{\mathrm{a}}$, Alessandro Giovinazzo ${ }^{\mathrm{b}}$, Francesca R. Bertani $^{\mathrm{c}}$, Luca Businaro $^{\mathrm{c}}$, Paolo \\ Bisegna $^{\mathrm{a}}$, Claudia Matteucci ${ }^{\mathrm{b}}$, Federica Casellia,* \\ ${ }^{a}$ Department of Civil Engineering and Computer Science, University of Rome Tor Vergata, 00133 Rome, Italy \\ ${ }^{b}$ Department of Experimental Medicine, University of Rome Tor Vergata, 00133 Rome, Italy \\ ${ }^{c}$ Institute for Photonics and Nanotechnologies, Italian National Research Council, 00156 Rome, Italy
}

\begin{abstract}
The study and the characterization of cell death mechanisms are fundamental in cell biology research. Traditional death/viability assays usually involve laborious sample preparation and expensive equipment or reagents. In this work, we use electrical impedance spectroscopy as a label-free methodology to characterize viable, necrotic and apoptotic human lymphoma U937 cells. A simple three-electrode coplanar layout is used in a differential measurement scheme and thousands of cells are measured at high-throughput $(\approx 200 \mathrm{cell} / \mathrm{s})$. Tailored signal processing enables accurate and robust cell characterization without the need for cell focusing systems. The results suggest that, at low frequency (0.5 MHz), signal magnitude enables the discrimination between viable/necrotic cells and cell fragments, whereas phase information allows discriminating between viable cells and necrotic cells. At higher frequency (10 MHz) two subpopulations of cell fragments are distinguished. This work substantiates the prominent role of electrical impedance spectroscopy for the development of next-generation cell viability assays.
\end{abstract}

Keywords: single-cell analysis, cell viability, microfluidic impedance spectroscopy, coplanar electrodes, flow cytometry

\section{Introduction}

The study and the characterization of cell death mechanisms are fundamental to unravel cell behavior in normal and harsh conditions, with important implication to understand disease pathogenesis and treatment. Apoptosis is a physiological form of programmed cell death whereby a cell provokes its own demise in response to a stimulus, whereas necrosis occurs when cells are irreversibly damaged by an external trauma. A critical issue in chemotherapy drugs development is the type of resulting cell death, both for the containment of inflammatory or immunosuppressive response, and to avoid the mechanism of resistance to the therapy (Zhang et al., 2014).

Cell death characterization is essential in cell biology research, and specific recommendations have been drawn up for the classification of the different types and modality of cell death discovered so far (Galluzzi and et al., 2018). Traditional death/viability assays involve

${ }^{*}$ Corresponding author. Email: caselli@ing.uniroma2.it laborious sample preparation and expensive equipment or reagents (Cummings et al., 2012). In particular, flow cytometry (Shapiro, 2003) predominantly relies on fluorescent labeling, which may be time-consuming and alter natural biological functions (Hiramatsu et al., 2019). Microfluidic lab-on-a-chip solutions promise simpler procedures and greatly reduced costs (Valero et al., 2005; Donald et al., 2010; Petchakup et al., 2017; Reale et al., 2019), with tremendous benefit for pharmaceutical and life-science research. In particular, labelfree approaches are especially attractive, because they preserve cell native behaviour and therapeutic potential (Rollo et al., 2017; Bagnaninchi and Drummond, 2011).

Since changes in cell physiology are known to result in changes in dielectric properties of the cell (Nikolic-Jaric et al., 2013), lab-on-a-chip devices for cell electrical phenotyping have the potential for labelfree death/viability assays. Wang et al. (2002) showed changes in the dielectric properties of HL-60 and Jurkat cells during apoptosis, by means of dielectrophoresis (DEP) crossover frequency measurements. Lee et al. (2009) reported differences between TE2 apoptotic and 
necrotic cells positioned between two electrodes, by time-lapse capacitance cytometry. Nikolic-Jaric et al. (2013) observed the onset of apoptosis in Chinese hamster ovary cells by using DEP-induced translation of flowing cells, at a throughput of about 1 cell/s.

Microfluidic impedance spectroscopy (Cheung et al., 2010; Sun and Morgan, 2010), potentially enabling high-throughput multiparametric analysis, has also been considered to perform viability assays. In a typical impedance chip, suspended particles flow through a microchannel and two electrode pairs are used to measure the variation in channel impedance induced by the passage of a particle. The impedance variation is exploited to characterize particle properties. Both coplanar and facing electrode configurations have been proposed in the literature. However, whereas fabricating facing electrodes requires advanced fabrication equipment and pose alignment issues, coplanar electrodes can be easily patterned at very small dimensions yielding miniaturized, reproducible, and ultimately low-cost devices (Hong et al., 2005; Xie et al., 2017; GonzálezMurillo et al., 2018). Gou et al. (2011) designed a T-shape microchannel with two coplanar electrodes to measure differences in resistance and capacitance of flowing SMMC-7721 cells at normal, apoptotic and necrotic status. Optical images where needed to detect necrotic particles, and the throughput of the system was below 1 cell/s. Kirkegaard et al. (2014) measured differences in electrical opacity between untreated and paclitaxel-treated HeLa cells, at a throughput of about 3 cell/s, by using a three-electrode coplanar configuration.

Unfortunately, resolution and accuracy of microfluidic impedance spectroscopy is challenged by the positional dependence of the measured signals, i.e. identical particles flowing along different trajectories in the microchannel provide different signals (Cheung et al., 2010; Spencer and Morgan, 2011; Caselli et al., 2018). This is due to the non-uniformity of the electric field in the sensing region (Sun et al., 2007; Riordon et al., 2012) and produces blurring of estimated particle properties (Spencer et al., 2016; Errico et al., 2017; Reale et al., 2018). An approach to cope with the positional dependence of the measured traces is particle focusing, for example by means of sheath flow (Mao et al., 2012), inertial focusing (Di Carlo, 2009) or active focusing mechanisms (Holmes et al., 2006; Shaker et al., 2014; Grenvall et al., 2014). However, they increase the complexity of the system and of its control. Another approach is to use microfluidic channels with crosssectional dimensions smaller than the diameter of the cell under investigation (Xie et al., 2017; Zhao et al., 2018). However, systems with a constriction structure are difficult to optimize for size-heterogeneous samples.

In this work, we address the label-free discrimination of viable, necrotic and apoptotic human lymphoma U937 cells in a microfluidic impedance cytometer. The U937 cell line (Sundström and Nilsson, 1976) is widely used in cell biology research, however its electrical phenotyping has received limited attention. In order to keep the system as simple as possible, thus maximizing its potential for low-cost and portable implementation, a chip layout with three coplanar electrodes is used and no focusing mechanisms are implemented. Due to the presence of both intact cells and apoptotic bodies, size-heterogeneous samples are expected. Accordingly, position blurring cannot be easily eliminated by optimization of channel dimensions. In order to mitigate the positional dependence of the measured signals, an enhanced version of the compensation strategy previously introduced by Errico et al. (2017) is used. In that paper, the approach was demonstrated for polystyrene beads and yeast cells sizing, using the real part of the impedance signal at low frequency. Here we exploit that methodology for the impedance profiling of human lymphoma cells. To this aim, the complex impedance traces at two frequencies are used and an ad-hoc signal processing is developed, enabling compensation on both signal amplitude and phase. Experimental campaigns involving control samples and samples treated with heat-shock (to induce necrosis) or etoposide (to induce apoptosis) are performed. Thousands of cells are measured at high-throughput $(\approx 200$ cell/s) and flow cytometry analysis is used for validation. The experimental protocol is pictured in Figure S1 of the Supplementary Material and detailed in Section 2, whereas a comparison with other relevant studies reported in the literature is provided in Table T1 of the Supplementary Material. The results of the present work (Section 3) show that the recorded electrical fingerprints provide highcontent information useful for cell discrimination, and bring microfluidic impedance spectroscopy in a prominent position for next-generation cell viability assays.

\section{Materials and methods}

\subsection{Working principle of the microfluidic impedance cytometer}

A schematic representation of the microfluidic impedance chip is shown in Figure 1(a). The microchannel is filled with a conductive fluid and a multifrequency AC voltage signal is applied to the central electrode by an impedance spectroscope. The difference in current collected from the lateral electrodes 
(a)

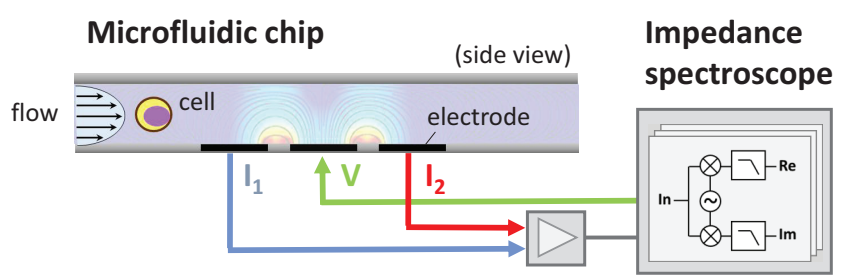

TA

(c)

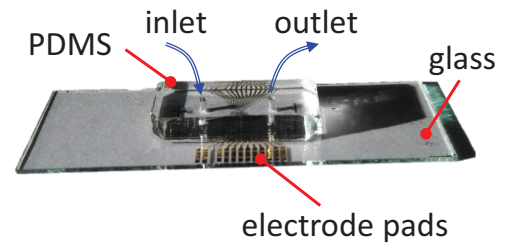

(b)
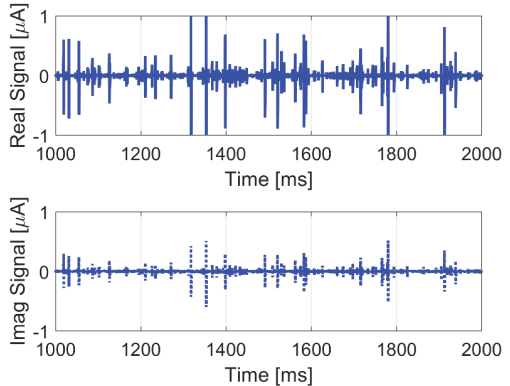

(e)

Figure 1: Microfluidic impedance spectroscopy. (a) Operation mode: an impedance spectroscope provides a multifrequency AC voltage signal ( $V$ ) to the central electrode of the microfluidic chip, and receives as differential input the current signals $I_{1}$ and $I_{2}$ collected from the lateral electrodes and conditioned by a transimpedance amplifier (TA). (b) Real and imaginary parts of the differential current demodulated at $0.5 \mathrm{MHz}$ (exemplary datastream shown). (c) Microfluidic impedance chip. (d) Fluidic layout (top view). (e) Bright-field image of the sensing region (top view, channel height is $21 \mu \mathrm{m})$.

is measured with a transimpedance amplifier and demodulated by the spectroscope, providing the in-phase (real) and out-of-phase (imaginary) components of each frequency channel. A portion of a typical datastream (relevant to one frequency channel) is reported in Figure 1(b). The passage of a flowing particle is recorded as a pair of opposite peaks (Sun et al., 2009). Due to the positional dependence issue, peak amplitude both depends on particle properties and particle trajectory height (with particles traveling near the electrodes providing higher peak amplitude than identical particles traveling far from the electrodes, cf. Figure S3 of the Supplementary Material). As demonstrated by Errico et al. (2017), the ratio between peak width and peak-topeak time, called shape parameter, correlates with particle trajectory height. Therefore, it can be used to purge out position blurring from the measured signals (cf. Section 2.5).

\subsection{Chip microfabrication}

The microfluidic impedance chip consists of a microchannel with coplanar microelectrodes integrated at the bottom of a microscope glass slide (Figure 1(c)(e)). The fluidic network was fabricated in polydimethylsiloxane (PDMS), following well-established replica molding procedures. The $\mathrm{Ti} / \mathrm{Au}$ electrodes $(20 \mathrm{~nm} / 200 \mathrm{~nm})$ were lithographically patterned onto the glass substrate $(75 \times 25 \times 1 \mathrm{~mm})$ by liftoff technique.
PDMS channel and electrodes were aligned and the chip was sealed after $\mathrm{O}_{2}$ plasma activation. In the sensing region the channel cross-sectional area is $40 \mu \mathrm{m}$ wide and $21 \mu \mathrm{m}$ high; electrodes are $30 \mu \mathrm{m}$ wide with a $10 \mu \mathrm{m}$ spacing. Arrays of micro-pillars were included at the inlet fluidic port to reduce the risk of channel clogging. A custom chip holder was used for fluidic and electric connections.

\subsection{Cell preparation and culture}

Human histiocytic lymphoma U937 cells, originally obtained from Zooprofilactic Institute (Brescia, Italy), were grown in suspension culture at a density of $4 \times 10^{5}$ cells/ml in RPMI 1640 supplemented with $10 \%$ fetal bovine serum (FBS), $2 \mathrm{mM}$ glutamine, $50 \mathrm{U} / \mathrm{ml}$ penicillin and $50 \mathrm{U} / \mathrm{ml}$ streptomycin (all reagents from Sigma-Aldrich, St Louis, MO, USA) at $37^{\circ} \mathrm{C}$ in a $5 \%$ $\mathrm{CO}_{2}$-humified atmosphere for $18 \mathrm{~h}$. To induce necrosis, after $15 \mathrm{~h}$ of culture cells were exposed to a heat shock by incubation for $30 \mathrm{~min}$ at $60{ }^{\circ} \mathrm{C}$ in a water bath, then washed in phosphate-buffered saline (PBS; Sigma-Aldrich) for $5 \mathrm{~min}$ at $1000 \mathrm{~g}$, and incubated in culture medium for other $3 \mathrm{~h}$ at $37{ }^{\circ} \mathrm{C}$ in a $5 \%$ $\mathrm{CO}_{2}$-humified atmosphere. Apoptosis was induced with etoposide (Sigma-Aldrich) treatment at the concentration of $50 \mu \mathrm{M}$ for $18 \mathrm{~h}$. Dimethyl sulfoxide (DMSO; Sigma-Aldrich) was used as diluent for etoposide and was referred as vehicle in control condition. Following 
incubation in the various culture conditions above described, the untreated and treated cells were harvested and washed three times with PBS for $5 \mathrm{~min}$ at $1000 \mathrm{~g}$ and filtered with $20 \mu \mathrm{m}$ filters (CellTrics) before successive manipulations. The trypan blue dye exclusion test was performed on each sample. A mixed sample, containing cells from the control, heat-shock and etoposide samples, was also prepared. Each sample was spiked with polystyrene beads ( $6 \mu \mathrm{m}$ diameter, PolySciences) for internal reference.

\subsection{Impedance data acquisition}

Prior to impedance data collection, the microfluidic chip was flushed with a $1 \%$ BSA (bovine serum albumin) solution in PBS to prevent cells adhesion to the PDMS walls. The sample was injected into the microfluidic chip by a syringe pump (Harvard Apparatus) at a rate of $10 \mu \mathrm{l} / \mathrm{min}$. The measurements were carried out at two simultaneous frequencies ( 0.5 and $10 \mathrm{MHz}, 2.5 \mathrm{~V}$ each) with an HF2TA transimpedance amplifier (Zurich Instruments, $10 \mathrm{k} \Omega$ gain) and a HF2IS impedance spectroscope (Zurich Instruments, $20 \mathrm{kHz}$ filter bandwidth, $115 \mathrm{kSa} / \mathrm{s}$ readout rate). A trypan blue dye exclusion test confirmed that the electric field in the sensing region had no adverse effects on the cells (cf. Figure S2 of the Supplementary Material).

\subsection{Impedance data processing}

Event detection in the data stream was performed with the algorithm described by Caselli and Bisegna (2016). For feature extraction, a novel template fitting strategy was implemented, taking into account all the complex frequency channels at the same time. To this aim, a suited bipolar Gaussian template was considered, characterized by the following features: a frequency-dependent complex amplitude $a$, a frequency-independent real peak-width control $\sigma$, and a frequency-independent real peak-to-peak time $\delta$ (cf. Figure S4 of the Supplementary Material). A leastsquare approach was used to fit the template to each detected event.

The following features were considered for discrimination:

- electrical diameter $D=G\left|a_{0.5}\right|^{1 / 3}$, where $a_{0.5}$ denotes complex amplitude at $0.5 \mathrm{MHz},|\cdot|$ denotes absolute value, and $G$ is a gain factor accounting for the electronic circuitry;

- phase of $a$ at $0.5 \mathrm{MHz}$, denoted by $\Phi_{0.5}$;

- phase of $a$ at $10 \mathrm{MHz}$, denoted by $\Phi_{10}$.
The shape parameter $\sigma / \delta$ of the signal pulse, correlating with the particle trajectory height, was also computed. An approximately linear relationship was found between each electrical feature and the shape parameter, thus enabling the implementation of a compensation procedure able to mitigate position blurring. In particular, novel formulas for compensation of electrical phases have been implemented (cf. Figure S5 of the Supplementary Material). Moreover, since the signal phase depends on a number of factors related to the physical properties of the complete system (Haandbæk et al., 2016; Rollo et al., 2017), the phase features $\Phi_{0.5}$ and $\Phi_{10}$ were normalized by subtracting their average value computed for the bead population, so they have the meaning of phase shift with respect to beads.

\subsection{High-speed image acquisition}

For check purposes, the sample flow through the detection area was acquired using a high-speed camera (Photron Mini UX100, frame rate $4000 \mathrm{fps,} \mathrm{shut-}$ ter time $3.9 \mu \mathrm{s}$ ) connected to an inverted microscope (Zeiss Axio Observer, 40× objective). Events detected in the impedance datastream were matched to their optical frames with a custom Matlab script.

\subsection{Flow cytometry data acquisition and processing}

For validation and comparison purposes, a multiparametric flow cytometry analysis, combining scatter plot and DNA labeling analysis, was performed to distinguish apoptotic, necrotic or viable cells (Matteucci et al., 1999). To this aim, harvested cells were fixed with $70 \%$ ethanol for $45 \mathrm{~min}$ at $4^{\circ} \mathrm{C}$, washed in PBS and stained with propidium iodide (PI) and RNase as previously described (Argaw-Denboba et al., 2017). Samples were analyzed by flow cytometry using a FACScan cytometer (BD Biosciences, San Jose, CA, USA). Detectors and amplifier gains for forward and orthogonal scatter were adequately selected to simultaneously detect viable, apoptotic and necrotic cells. Data acquisition and analysis were performed using CellQuest software on a minimum of 5000 events up to 10000 for each sample (BD Biosciences).

\section{Results and discussion}

\subsection{Flow cytometry characterization}

By a multiparametric analysis, flow cytometry allows to distinguish apoptotic, necrotic or viable cells combining the morphological features together with the detection of hypodiploid DNA fragmentation typical of the apoptotic cell (Matteucci et al., 1999). The scatter 

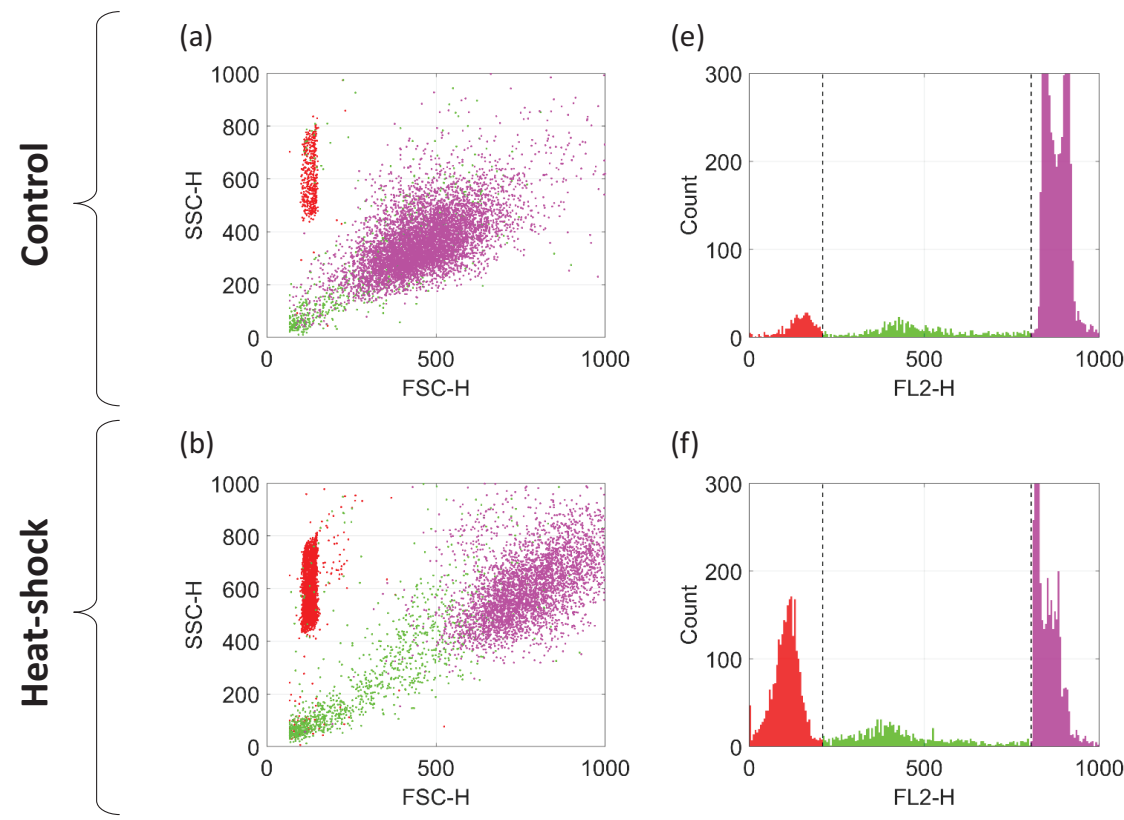

(f)
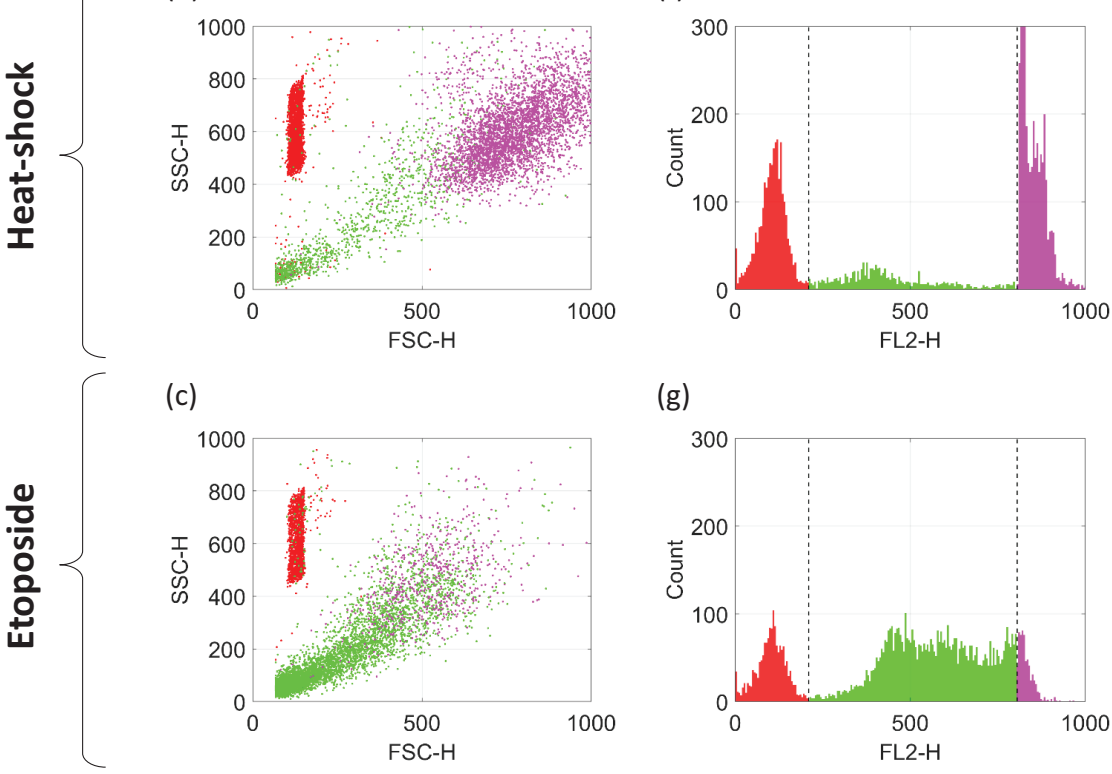

(g)
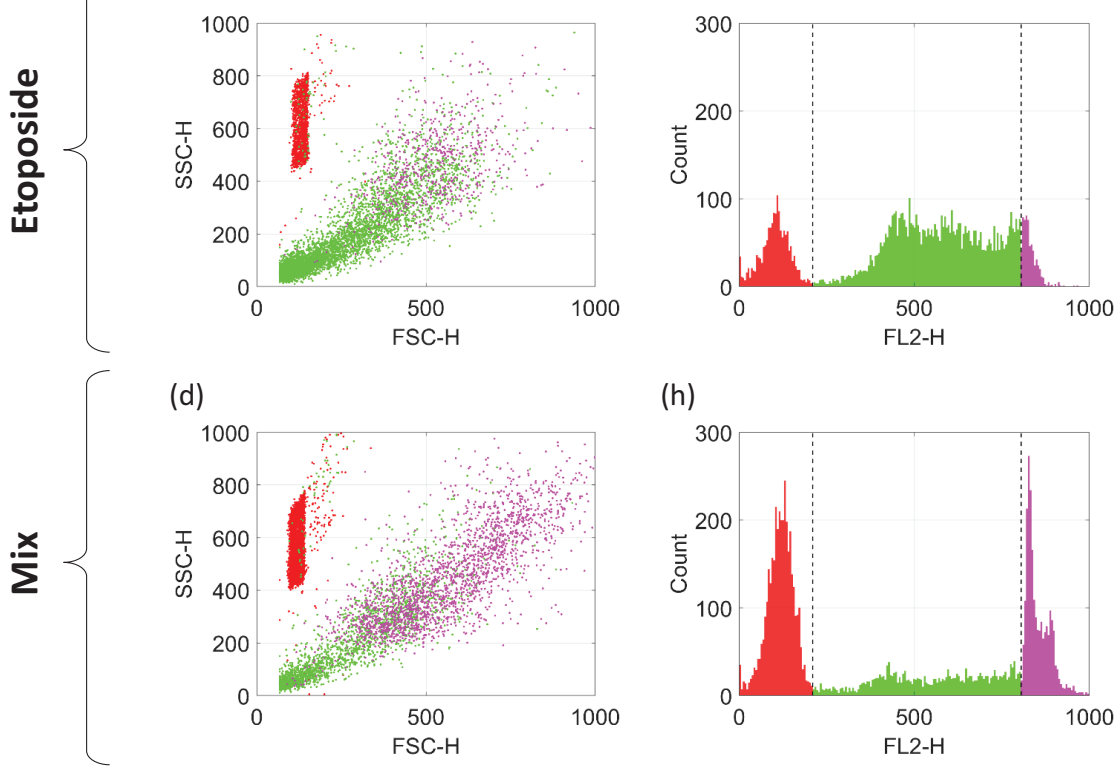

(d)

(h)
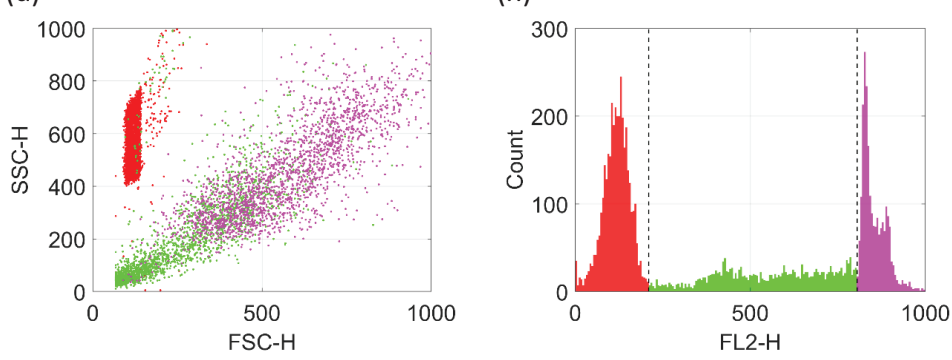

Figure 2: Flow cytometry analysis of in vitro treated U937 cells (first row, control sample; second row, heat-shock treatment; third row, etoposide treatment; fourth row, mixed sample). (a-d) Scatter plots of the side scatter height (SSC-H) vs. the forward scatter height (FSC-H). (e-f) Histograms of the fluorescence 2 height (FL2-H) channel value. Populations are colour-labeled according to their FL2-H value. 
plot of the forward-scatter height (FSC-H) versus sidescatter height (SSC-H) parameters, and the histogram of the events according to fluorescence 2 height (FL2$\mathrm{H})$ channel values are reported in Figure 2, for each experimental condition. FSC-H versus SSC-H analysis was performed to assess the morphological features (Adan et al., 2017). A gating FSC-H $\geq 70$ was applied. The markers on FL2-H histograms (panels e-h) were set according to the PI content, generating gates related to cells containing diploid and hyperdiploid DNA (pink), hypodiploid DNA (green) or debris/beads (red). Correspondingly, the scatter plots (panels a-d) show the distribution of the different cell populations according to the DNA content. Although viable and heatshock treated cells retain the same FL2-H profile in diploid/hyperdiploid DNA content (panels e and f), the necrotic cells show a different distribution in the scatter plot (panels a and b; pink). Otherwise, most of the cells treated with etoposide present the typical emission of hypodiploid DNA (panel g), as expected for apoptotic cells, and correspond to a low FSC/SSC scatter plotting (panel c; green). Analysis of samples containing a mix of cells from the different preparations (panels $d$ and $h$ ) overlaps the results obtained by the single sample analysis. Particularly, signals from diploid and hyperdiploid DNA (panel h; pink) correspond to two clusters of cells in the scatter plot (panel d; pink) most probably derived from both living and necrotic cells. As expected, signals from hypodiploid DNA (panel h; green) correspond to a low FSC/SSC scatter plotting (panel d; green). Gating of flow cytometry data is shown in Figure S7 of the Supplementary Material.

\subsection{Impedance-based characterization}

A gallery of exemplary event signals acquired by microfluidic impedance spectroscopy, relevant to the mixed sample, is shown in Figure 3. For each event, the real and imaginary parts of the differential current signals demodulated at $0.5 \mathrm{MHz}$ and $10 \mathrm{MHz}$ are shown, along with a snapshot of the relevant flowing particle. By looking at the real part of the low frequency signal (blue solid line), significant differences in peak amplitude are noticed, with some events (panels b and c) exhibiting an amplitude similar to that of a bead (panel a), and some events (panels d and e) with larger signal amplitude. This is reflected by differences in the electrical diameter $D$. For each frequency channel, differences in the ratio between the peak amplitudes of the imaginary (dotted line) and real (solid line) signal components are also evident. This is reflected by differences in the phases $\Phi_{0.5}$ and $\Phi_{10}$.
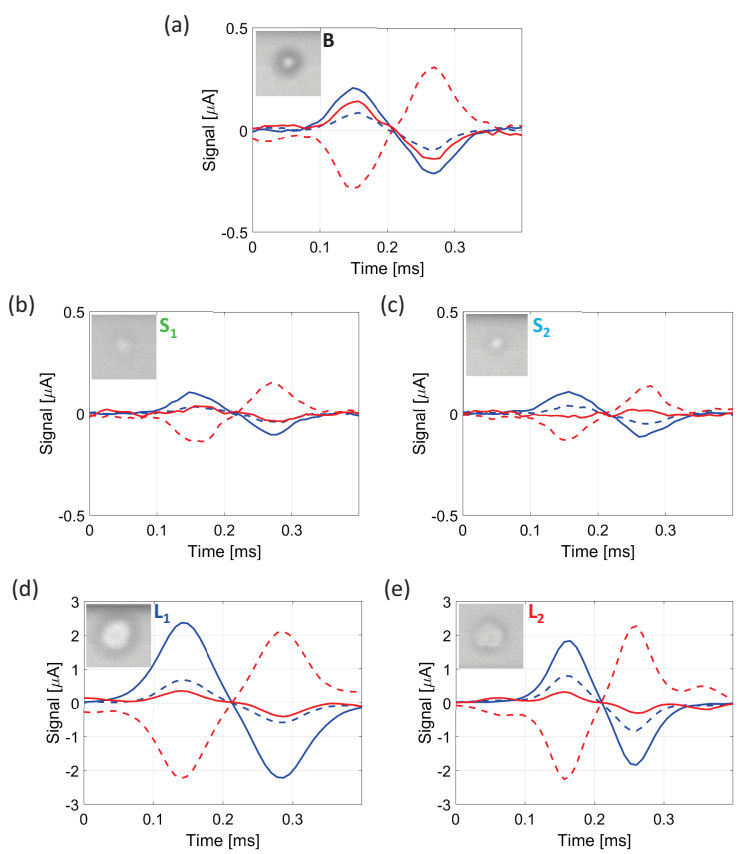

(e)

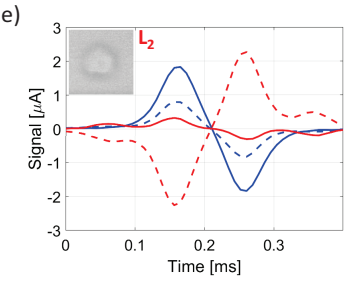

Figure 3: Gallery of exemplary event signals. Real (solid line) and imaginary (dotted line) part of demodulated differential current signal measured at $0.5 \mathrm{MHz}$ (blue lines) or $10 \mathrm{MHz}$ (red lines). A snapshot $(100 \times 100$ pixels $)$ of the corresponding flowing particle is also shown. As detailed in Section 3.2, the five examples exhibit different electrical features and the relevant particles belong to different bead/cell populations (namely, $\mathrm{B}, \mathrm{S}_{1}, \mathrm{~S}_{2}, \mathrm{~L}_{1}$, and $\mathrm{L}_{2}$, cf. Figure 4 and the relevant discussion). 


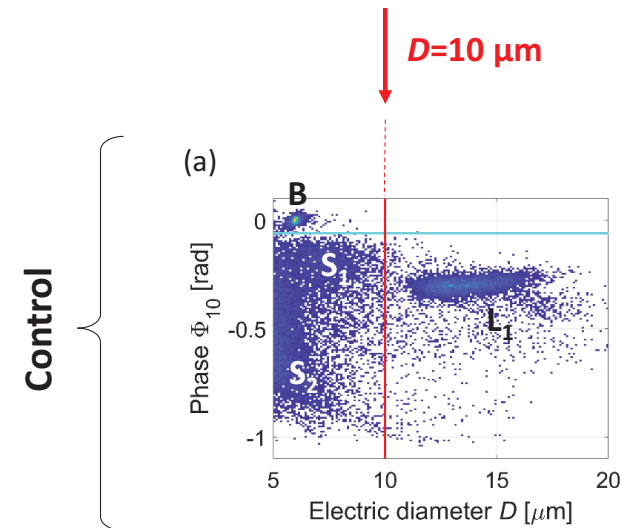

(b)

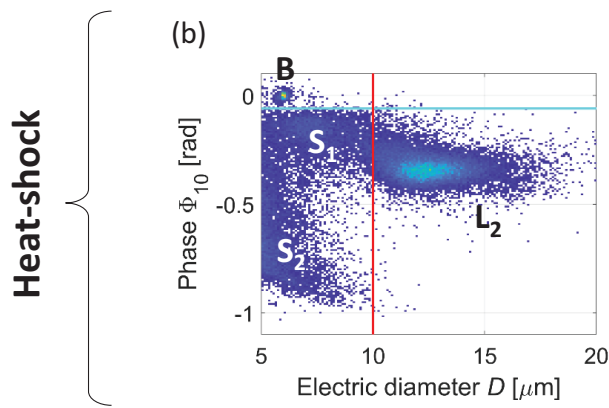

(c)

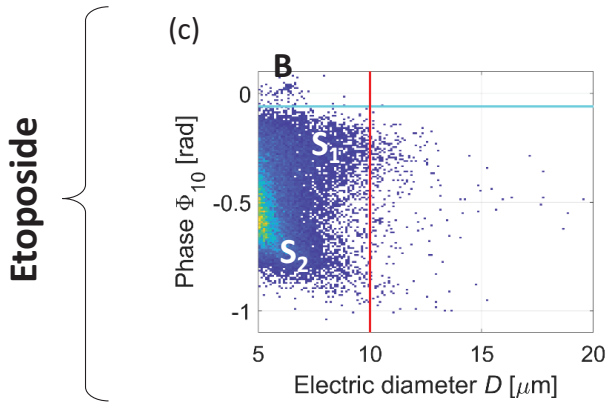

(d)

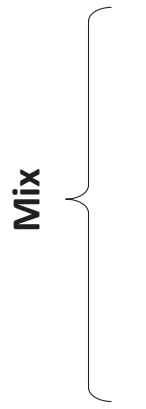

Small electric diameter

$(D<10 \mu \mathrm{m})$

(e)

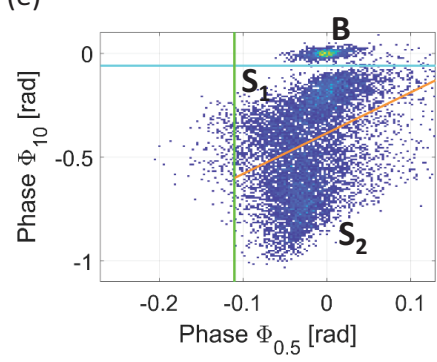

(f)

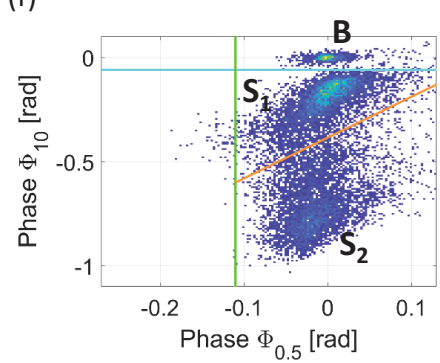

(g)

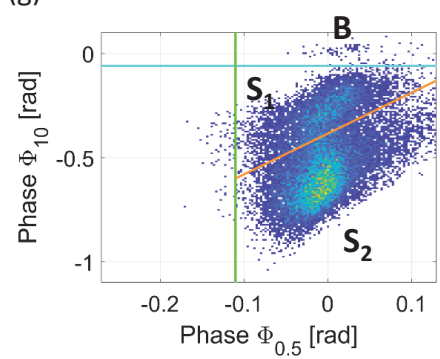

(h)

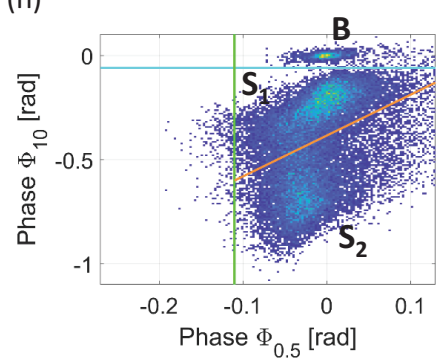

Large electric diameter

$(D>10 \mu \mathrm{m})$

(i)

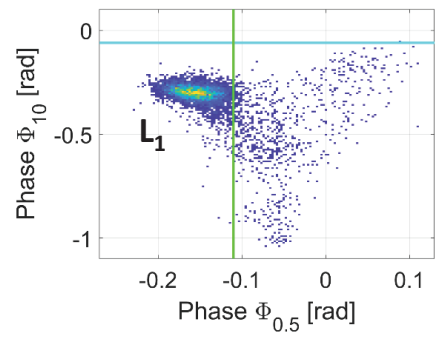

(j)

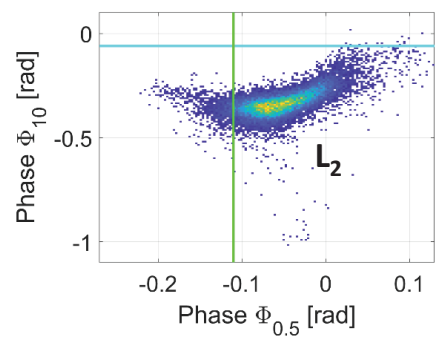

(k)

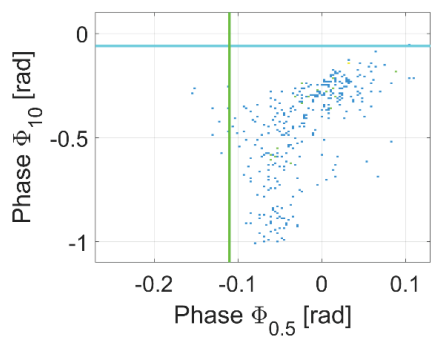

(I)

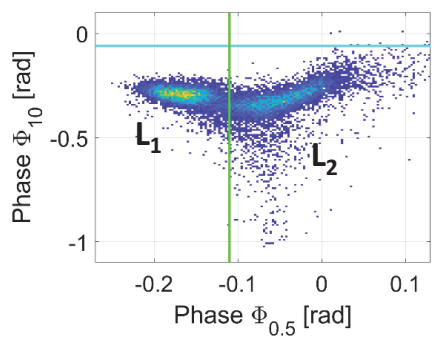

Figure 4: Impedance spectroscopy analysis results (first row, control sample; second row, heat-shock treatment; third row, etoposide treatment; fourth row, mixed sample). (a-d) Density plots of phase at $10 \mathrm{MHz}$ vs. electrical diameter. (e-h) Phase at $10 \mathrm{MHz}$ vs. phase at $0.5 \mathrm{MHz}$ with a gating on small events $(D<10 \mu \mathrm{m})$. (i-1) Phase at $10 \mathrm{MHz}$ vs. phase at $0.5 \mathrm{MHz}$ with a gating on large events $(D>10 \mu \mathrm{m})$. 
By using the compensation procedure described in Section 2.5, a significant mitigation of position blurring was obtained (cf. Figures S5 and S6 of the Supplementary Material).

The impedance-based signatures of the three experimental conditions (control, heat-shock treatment, and etoposide treatment) are reported in Figure 4 (rows one to three, respectively). Events with an electrical diameter $D$ lower than $5 \mu \mathrm{m}$ were discarded from the analysis, in agreement with the gating used in flow cytometry acquisition. In each experimental condition, at least 15000 events were considered. The density plots of the high-frequency phase $\Phi_{10}$ against the electrical diameter $D$ (panels a-c) reveal different event clusters spanning a wide range of cell electrical diameters. The bead population (B) is easily identified in the upper left region. All other data points exhibit a negative highfrequency phase shift (cf. horizontal cyan line). Following Petchakup et al. (2018), in order to facilitate the analysis, the events are divided into two groups based on electrical size (cf. vertical red line), i.e., events with small electrical diameter $(D<10 \mu \mathrm{m})$ and events with large electrical diameter $(D \geq 10 \mu \mathrm{m})$. The density plots of the high-frequency phase $\Phi_{10}$ against the lowfrequency phase $\Phi_{0.5}$ are shown in panels e-g and panels $\mathrm{i}-\mathrm{k}$ for the events with small and large electrical diameter, respectively. Besides the beads, in all the experimental conditions two populations of small events are identified, labeled as $S_{1}$ and $S_{2}$ (cf. panels e-g; oblique orange line). Concerning the large events, one welldefined cluster is evident in panel i (control sample, cluster $\mathrm{L}_{1}$ ) and in panel $\mathrm{j}$ (heat-shock treatment, cluster $L_{2}$ ). Those clusters $L_{1}$ and $L_{2}$ exhibit different values of low-frequency phase $\Phi_{0.5}$ (cf. vertical green line). None of them is found in case of etoposide treatment (panel $\mathrm{k})$.

Analysis of the sample containing a mix of cells from the different preparations (Figure 4, fourth row) overlaps the results obtained by the single sample analysis. Particularly, besides the beads (B), the two clusters with small electric diameters $\left(S_{1}\right.$ and $\left.S_{2}\right)$ and the two clusters with large electric diameter $\left(\mathrm{L}_{1}\right.$ and $\left.\mathrm{L}_{2}\right)$ are found (cf. panel $\mathrm{h}$ and panel 1 , respectively). Figure 5(a) shows the localization of those clusters in a $3 \mathrm{D}$ scatter plot of the electrical features $D, \Phi_{0.5}$ and $\Phi_{10}$.

A video correlating recorded impedance data with captured optical images is provided as Supplementary Material. In particular, the video shows a total of fifteen events drawn from the analysis of the mixed sample, three events for each of the five populations $\left(B, S_{1}, S_{2}\right.$, $\mathrm{L}_{1}$, and $\mathrm{L}_{2}$ ). For each event, images of the cell/particle flowing through the microfluidic chip are shown. The impedance signals generated by the flowing cell/particle are also visualized, and the corresponding values of the electric phases $\Phi_{0.5}$ and $\Phi_{10}$ are marked in the relevant density plot. An exemplary video frame is reported in Figure S8 of the Supplementary Material.

In order to assess the reproducibility of the results, the experimental campaign was repeated four times, across a time frame of six months, using different microfluidic impedance chips. In all repetitions, the appearance and location of the clusters $\mathrm{L}_{1}, \mathrm{~L}_{2}, \mathrm{~S}_{1}$, and $\mathrm{S}_{2}$ was confirmed. Figure 5(b)-(e) shows the centroid values of the electrical phases at low frequency $\left(\Phi_{05}\right.$; panels b and c) and high-frequency $\left(\Phi_{10}\right.$; panels $\mathrm{d}$ and e) for the different clusters. In particular, cluster $\mathrm{L}_{1}$ of the control sample and cluster $\mathrm{L}_{2}$ of the heat-shock sample are considered in panels $b$ and $d$, whereas clusters $S_{1}$ and $S_{2}$ of the etoposide sample are considered in panels $\mathrm{c}$ and e. The error bar relevant to the four repetitions (mean \pm standard deviation) is also shown. Cluster $\mathrm{L}_{1}$ of the control sample and cluster $\mathrm{L}_{2}$ of the heat-shock sample exhibited a significantly different electric phase at low frequency $(\mathrm{P} \leq 0.001)$. Moreover, clusters $\mathrm{S}_{1}$ and $\mathrm{S}_{2}$ of the etoposide sample exhibited a significantly different electric phase at high frequency $(\mathrm{P} \leq 0.001)$. Other differences were not significant.

\subsection{Discussion}

Canonical hallmarks of apoptotic cell death include cell shrinkage, maintenance of plasma membrane integrity, nuclear fragmentation and formation of apoptotic bodies. In contrast, necrosis is characterized, among other features, by an early swelling and increased permeability with break-down of the plasma membrane integrity. Accordingly, different size and dielectric properties are expected for apoptotic, necrotic and viable cells, and this should reflect in different impedance signatures. The influence of cell geometric and dielectric properties on measured impedance signals is briefly reviewed in Section 6 of the Supplementary Material.

Herein, flow cytometry analysis and trypan blue exclusion test were used to characterize the different sample conditions and validate the results obtained by the impedance analysis. In fact, those methods confirmed the presence of DNA fragmentation in etoposide treated cells, of necrosis in heat-shock treated cells, and of mainly viable cells in control.

Viable U937 cells have a size greater than $10 \mu \mathrm{m}(\mathrm{Mi}$ et al., 2003). Since the electrical diameter $D$ is a metric of cell size (Gawad et al., 2004; Errico et al., 2017), this suggests that cluster $\mathrm{L}_{1}$ in the control sample (Figure 4, panels a and i) corresponds to viable cells. On the 


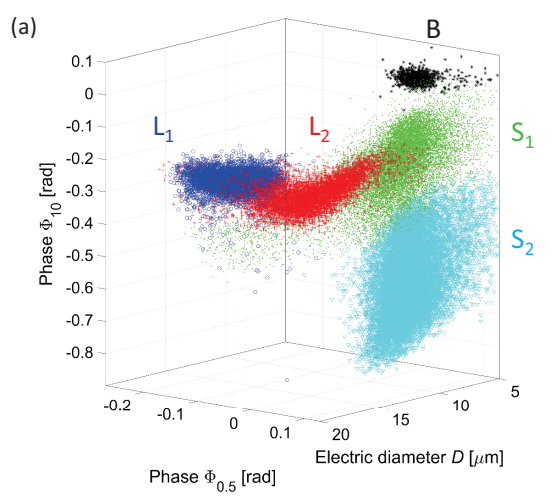

(b)
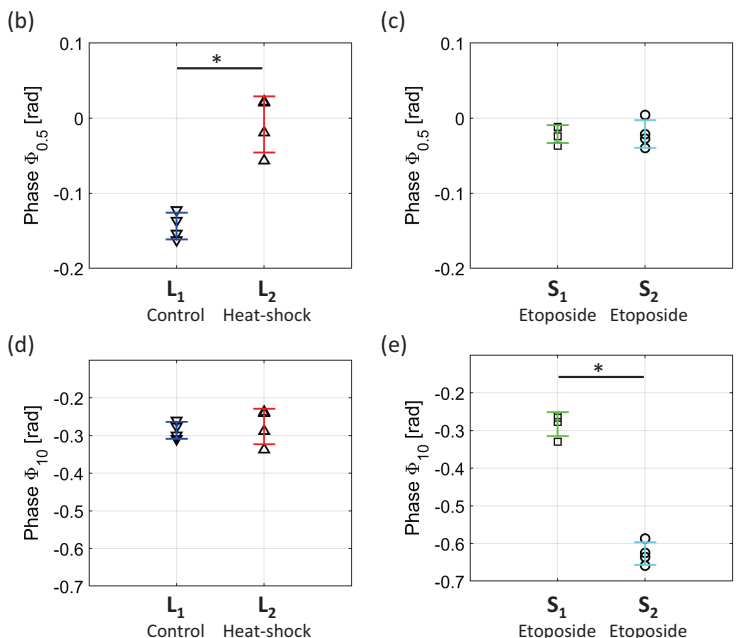

(e)

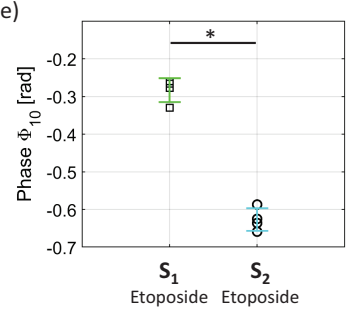

Figure 5: (a) 3D scatter plot of electrical diameter, phase at $0.5 \mathrm{MHz}$ and phase at $10 \mathrm{MHz}$, relevant to the mixed sample. Data points are labeled according to the population they belong to. (b-e) Impedancesignatures across four experiment repetitions. (b) and (d) respectively report the centroid value of the phases at low and high frequency $\left(\Phi_{0.5}\right.$ and $\Phi_{10}$ ), for the population $\mathrm{L}_{1}$ in the control sample, and the population $\mathrm{L}_{2}$ in the heat-shock sample. Bars were represented as mean \pm standard deviation, $* \mathrm{P} \leq 0.001$. (c) and (e) reports the analogous quantities for the populations $S_{1}$ and $S_{2}$ in the sample treated with etoposide. other hand, the lack of events with large electrical diameter in the sample treated with etoposide (Figure 4, panels $\mathrm{c}$ and $\mathrm{k}$ ) is in agreement with cell fragmentation into apoptotic bodies, whose dimension is smaller than that of the intact cell. This is also in agreement with the result of flow cytometry analysis for the sample treated with etoposide, where the majority of events had hypodiploid DNA (as expected for apoptotic cells) and were located in the low FSC region (Figure 2, panels $\mathrm{c}$ and $\mathrm{g}$ ).

A cluster of cells with large electrical diameter $\left(\mathrm{L}_{2}\right)$ is present in the sample treated with heat shock, that is known to induce cell necrosis. This cluster exhibits a different low-frequency phase $\Phi_{0.5}$ with respect to cluster $\mathrm{L}_{1}$ of the control sample. Since the electric phase depends on cell membrane properties (Pierzchalski et al., 2010), this suggests that cluster $\mathrm{L}_{2}$ corresponds to cell with altered plasma membrane, most probably necrotic cells.

Events with small electrical diameter are present in all the experimental conditions, although in different proportions with respect to the events with large electrical diameter. Similarly, an amount of events with hypodiploid DNA mapping into the low FSC/SSC region is found at flow cytometry analysis in each experimental condition (Figure 2; green). Those events presumably correspond to apoptotic bodies and cellular debris. An analysis essentially based on the highfrequency phase $\Phi_{10}$ allows to distinguish two different populations, namely clusters $S_{1}$ and $S_{2}$. Cluster $S_{1}$ exhibits both low and high frequency phase signature close to that of cluster $\mathrm{L}_{2}$ (necrotic cells). As a perspective, tailored experimental campaigns will be devised in order to investigate the different subsets of cell-derived fragments, a topic which is gaining increasing attention in recent years (Jiang et al., 2017).

In order to further support the interpretation of the results provided by the impedance-based characterization, a simple model of the device was implemented (cf. Section 6 of the Supplementary Material). The simulated impedance spectra reported in Figure $S 9$ show that the electric diameter $D$ at $0.5 \mathrm{MHz}$ discriminates between $6 \mu \mathrm{m}$ diameter cells and $18 \mu \mathrm{m}$ diameter cells. Permeabilization of the cell membrane induces about $15 \%$ reduction of electric diameter. The electric phase at $0.5 \mathrm{MHz}, \Phi_{0.5}$, discriminates between intact and permeabilized $18 \mu \mathrm{m}$ diameter cells. In particular, intact [resp., permeabilized] cells exhibit negative [resp., nearly vanishing] phase shift with respect to beads. Analogously, the electric phase at $10 \mathrm{MHz}, \Phi_{10}$, discriminates between intact and permeabilized $6 \mu \mathrm{m}$ diameter cells. 
(b)

(a)

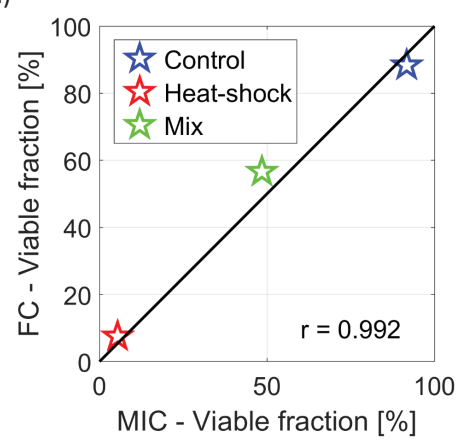

(d)
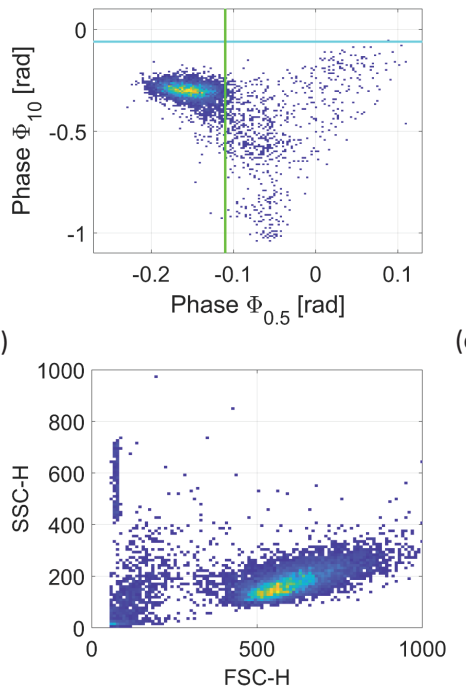

(c)

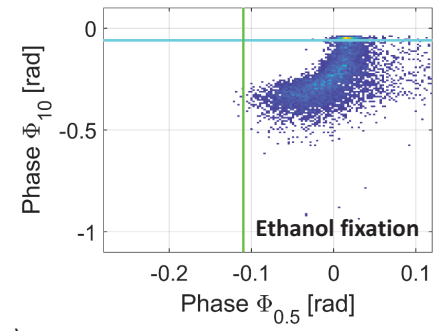

(e)

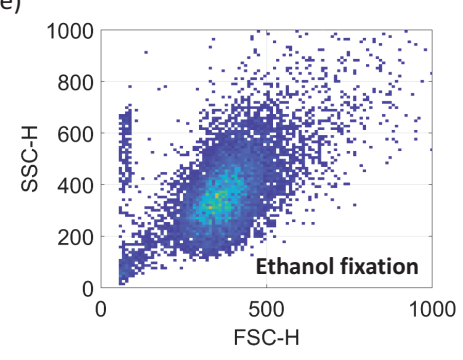

Figure 6: (a) Comparison between microfluidic impedance cytometry (MIC) and flow-cytometry (FC) analysis, in terms of the relative fraction of viable cells over large cells (i.e., viable and necrotic cells) in the control, heat-shock or mixed samples (correlation coefficient $r=0.992)$. (b-c) MIC analysis. Electrical phases of the control sample before (panel b) and after (panel c) ethanol fixation (gating $D>10 \mu \mathrm{m}$ ). (d-e) FC analysis. FSC-H and SSC-H parameters of the control sample before (panel d) and after (panel e) ethanol fixation.

Those simulation results are in agreement with the interpretation of clusters $\mathrm{L}_{1}$ and $\mathrm{L}_{2}$ as viable cells and necrotic cells, respectively. In fact, both clusters exhibit large electric diameter $D$, with $\mathrm{L}_{2}$ slightly shifted towards lower values (cf. Figure S6(d) of the Supplementary Material). Moreover, cluster $\mathrm{L}_{1}$ exhibits significantly lower phase $\Phi_{0.5}$ than cluster $\mathrm{L}_{2}$ (cf. Figure S6(e) and Figure 5(b)). Finally, the analysis of the simulated impedance spectra supports the interpretation of clusters $S_{1}$ and $S_{2}$ as two subpopulations of cell debris/apoptotic bodies, possibly characterized by damaged and intact membrane, respectively. In fact, both clusters exhibit small electric diameter $D$, with cluster $\mathrm{S}_{2}$ showing significantly lower phase $\Phi_{10}$ than cluster $\mathrm{S}_{1}$ (cf. Figure 5(e)).

Figure 6(a) compares the relative fraction of viable cells provided by impedance-based or flow-cytometry analysis, in the control, heat-shock and mixed samples. A good agreement is found (correlation coefficient $r=0.992$ ). As opposite to flow cytometry analysis, impedance-based analysis does not require any cell fixation and staining. Figure 6(b) and (c) compare the electrical phases $\left(\Phi_{0.5}\right.$ and $\left.\Phi_{10}\right)$ of the control sample before (panel b) and after (panel c) fixation with ethanol. Analogously, the morphological parameters provided by flow cytometry (FSC-H and SSC-H) before and after ethanol fixation are provided in Figure 6(d) and (e), respectively. It can be noticed that ethanol fixation sig- nificantly alters cell morphological and dielectric properties. In particular, electrical features similar to that of the heat-shock sample are recognized (cf. also Figure S10 of the Supplementary Material).

Diverse microfluidic impedance-based devices have been proposed in the literature as promising labelfree approaches for cell viability assays. Table T1 of the Supplementary Material provides an overview of systems based on simple coplanar-electrode configurations. The system presented in this work compares favourably with Gou et al. (2011), Kirkegaard et al. (2014), González-Murillo et al. (2018) in terms of throughput and discrimination capabilities, and with Xie et al. (2017) in terms of ability to deal with sizeheterogeneous samples.

Possible sources of error in microfluidic impedance cytometry include: i) classification/counting errors due to coincidences (i.e., two or more particles simultaneously present in the sensing zone), which pose an upper limit to sample concentration; ii) counting errors due to cell sedimentation inside the sample syringe and tubing, which can be minimized by using high flow rates or syringe stirrers; iii) classification/counting errors due to electronic noise in the measured data streams, which are minimized by means of robust signal processing routines. Other operative issues may arise from nonspecific cell adhesion to the channel walls, which is prevented by channel priming (e.g., with BSA solution), or 
channel clogging, which is alleviated by means of anticlogging pillars or sample filtering.

\section{Conclusions}

An impedance-based label-free analysis of viable, necrotic and apoptotic human lymphoma cells was reported. Necrosis was induced with heat-shock, whereas apoptosis was induced with etoposide. Electrical fingerprints from thousands of cells were measured and significant differences were found among untreated cells, cells treated with heat-shock, and cells treated with etoposide. Effect of ethanol fixation was also investigated. Overall, the results of the impedance-based analysis suggest that the electrical diameter at low frequency is effective for size-based discrimination between viable/necrotic cells and apoptotic bodies, whereas the electrical phase at low frequency is effective to discriminate between viable and necrotic cells based on intact or damaged plasma membrane.

Compared to other microfluidic impedance-based approaches, the proposed approach enjoys increased accuracy, thanks to mitigation of position-induced blurring, high-throughput and simplicity of the design (coplanar electrodes, no cell focusing mechanism needed). Accordingly, the system has the potential for integration into a compact low-cost device for cell viability assessment. Compared to flow cytometry analysis, impedance spectroscopy has the key advantage of minimal sample preparation (neither cell fixation nor fluorescent labeling are required). Moreover, its non-invasive nature is an asset when analysing cells intended for subsequent use (e.g., in cell therapy applications).

\section{Acknowledgements}

This work was supported by the Scientific Independence of Young Researchers Programme (SIR 2014) under Grant RBSI14TX20-MUSIC "Multidimensional Single-Cell Microfluidic Impedance Cytometry”.

\section{Appendix A. Supplementary data}

Supplementary data associated with this article can be found, in the online version, at http://dx.doi.org/10.1016/xxx.

\section{References}

Adan, A., Alizada, G., Kiraz, Y., Baran, Y., Nalbant, A., 2017. Critical Reviews in Biotechnology 37, 163-176. doi: $10.3109 / 07388551.2015 .1128876$.
Argaw-Denboba, A., Balestrieri, E., Serafino, A., Cipriani, C., Bucci, I., Sorrentino, R., Sciamanna, I., Gambacurta, A., SinibaldiVallebona, P., Matteucci, C., 2017. Journal of Experimental \& Clinical Cancer Research 36, 20. doi:10.1186/s13046-016-0485-

Bagnaninchi, P., Drummond, N., 2011. Proceedings of the National Academy of Sciences of the United States of America 108, 64626467. doi:10.1073/pnas.1018260108.

Caselli, F., Bisegna, P., 2016. IEEE Trans Biomed Eng 63, 415-422. doi:10.1109/TBME.2015.2462292.

Caselli, F., De Ninno, A., Reale, R., Businaro, L., Bisegna, P., 2018. Sensors and Actuators B: Chemical 256, 580-589. doi:10.1016/j.snb.2017.10.113.

Cheung, K.C., Di Berardino, M., Schade-Kampmann, G., Hebeisen, M., Pierzchalski, A., Bocsi, J., Mittag, A., Tárnok, A., 2010. Cytometry Part A 77, 648-666. doi:10.1002/cyto.a.20910.

Cummings, B.S., Wills, L.P., Schnellmann, R.G., 2012. Current Protocols in Pharmacology 56, 12.8.1-12.8.24. doi:10.1002/0471141755.ph1208s56.

Di Carlo, D., 2009. Lab Chip 9, 3038-3046. doi:10.1039/b912547g.

Donald, W., Joanna, S., Zbigniew, D., 2010. Cytometry Part A 77, 591-606. doi:10.1002/cyto.a.20889.

Errico, V., De Ninno, A., Bertani, F.R., Businaro, L., Bisegna, P., Caselli, F., 2017. Sensors and Actuators B: Chemical 247, 580586. doi:10.1016/j.snb.2017.03.035.

Galluzzi, L., et al., 2018. Cell Death and Differentiation 25, 486-541. doi:10.1038/s41418-017-0012-4.

Gawad, S., Cheung, K., Seger, U., Bertsch, A., Renaud, P., 2004. Lab Chip 4, 241-251. doi:10.1039/b313761a.

González-Murillo, J.J., Monge-Azemar, M., Bartoli, J., Flores, A., Moreno, M., Garca-Celma, M., Romano-Rodrguez, A., Svendsen, W., Samitier, J., Rodrguez-Trujillo, R., 2018. in: 2018 Spanish Conference on Electron Devices (CDE), pp. 1-4. doi:10.1109/CDE.2018.8597037.

Gou, H.L., Zhang, X.B., Bao, N., Xu, J.J., Xia, X.H., Chen, H.Y., 2011. J. Chromatogr. A 1218, 5725-5729. doi:10.1016/j.chroma.2011.06.102.

Grenvall, C., Antfolk, C., Bisgaard, C., Laurell, T., 2014. Lab Chip 14, 4629-4637. doi:10.1039/C4LC00982G.

Haandbæk, N., Bürgel, S.C., Rudolf, F., Heer, F., Hierlemann, A., 2016. ACS Sens 1, 1020-1027. doi:10.1021/acssensors.6b00286.

Hiramatsu, K., Ideguchi, T., Yonamine, Y., Lee, S., Luo, Y., Hashimoto, K., Ito, T., Hase, M., Park, J., Kasai, Y., Sakuma, S. Hayakawa, T., Arai, F., Hoshino, Y., Goda, K., 2019. Sci Adv. 5, eaau0241. doi:10.1126/sciadv.aau0241.

Holmes, D., Morgan, H., Green, N., 2006. Biosens. Bioelectron 21, 1621-1630. doi:10.1016/j.bios.2005.10.017.

Hong, J., Yoon, D.S., Kim, S.K., Kim, T.S., Kim, S., Pak, E.Y., No, K., 2005. Lab Chip 5, 270-279. doi:10.1039/B410325D

Jiang, L., Paone, S., Caruso, S., Atkin-Smith, G.K., Phan, T.K., Hulett, M.D., Poon, I.K.H., 2017. Scientific Reports 7, 14444. doi:10.1038/s41598-017-14305-z.

Kirkegaard, J., Clausen, C.H., Rodriguez-Trujillo, R., Svendsen, W.E., 2014. Biosensors 4, 257-272. doi:10.3390/bios4030257.

Lee, R.M., Choi, H., Shin, J.S., Kim, K., Yoo, K.H., 2009. Biosensors and Bioelectronics 24, 2586-2591. doi:10.1016/j.bios.2009.01.028.

Mao, X., Nawaz, A., Lin, S., Lapsley, M., Zhao, Y., McCoy, J., El-Deiry, W., Huang, T., 2012. Biomicrofluidics 6, 024113. doi:10.1063/1.3701566

Matteucci, C., Grelli, S., De Smaele, E., Fontana, C., Mastino, A., 1999. Cytometry 35, 145-153. doi:10.1002/(SICI)10970320(19990201)35:2;145::AID-CYTO6 $33.0 . C O ; 2-2$.

Mi, Y., Thomas, S.D., Xu, X., Casson, L.K., Miller, D.M., Bates, P.J., 2003. Journal of Biological Chemistry 278, 8572-8579. 
doi:10.1074/jbc.M207637200.

Nikolic-Jaric, M., Cabel, T., Salimi, E., Bhide, A., Braasch, K., Butler, M., Bridges, G., Thomson, D., 2013. Biomicrofluidics. 7. doi: $10.1063 / 1.4793223$.

Petchakup, C., Li, K.H.H., Hou, H.W., 2017. Micromachines 8. doi: $10.3390 / \mathrm{mi} 8030087$.

Petchakup, C., Tay, H.M., Yeap, W.H., Dalan, R., Wong, S.C., Li, K.H.H., Hou, H.W., 2018. Biosensors and Bioelectronics 118, 195203. doi:10.1016/j.bios.2018.07.052.

Pierzchalski, A., Hebeisen, M., Mittag, A., Berardino, M.D., Tarnok, A., 2010. in: Farkas, D.L., Nicolau, D.V., Leif, R.C. (Eds.), Imaging, Manipulation, and Analysis of Biomolecules, Cells, and Tissues VIII, International Society for Optics and Photonics. SPIE. pp. 256-266. doi:10.1117/12.840865.

Reale, R., De Ninno, A., Businaro, L., Bisegna, P., Caselli, F., 2018. Microfluidics and Nanofluidics 22, 41. doi:10.1007/s10404-0182055-3.

Reale, R., De Ninno, A., Businaro, L., Bisegna, P., Caselli, F., 2019. Lab Chip 19, 1818-1827. doi:10.1039/C9LC00071B.

Riordon, J., M. Catafard, N., Godin, M., 2012. Appl. Phys. Lett. 101, 154105. doi:10.1063/1.4759033.

Rollo, E., Tenaglia, E., Genolet, R., Bianchi, E., Harari, A., Coukos, G., Guiducci, C., 2017. Biosensors and Bioelectronics 94, 193199. doi:10.1016/j.bios.2017.02.047.

Shaker, M., Colella, L., Caselli, F., Bisegna, P., Renaud, P., 2014. Lab Chip 14, 2548-2555. doi:10.1039/c4lc00221k.

Shapiro, H., 2003. Practical Flow Cytometry, 4th Edition. Wiley.

Spencer, D., Caselli, F., Bisegna, P., Morgan, H., 2016. Lab Chip 16, 2467-2473. doi:10.1039/c6lc00339g.

Spencer, D., Morgan, H., 2011. Lab Chip 11, 1234-1239. doi:10.1039/c1lc20016j.

Sun, T., van Berkel, C., Green, N.G., Morgan, H., 2009. Microfluid. Nanofluid. 6, 179-187. doi:10.1007/s10404-008-0315-3.

Sun, T., Green, N.G., Gawad, S., Morgan, H., 2007. IET Nanobiotechnol. 1, 69-79. doi:10.1049/iet-nbt:20070019.

Sun, T., Morgan, H., 2010. Microfluid. Nanofluid. 8, 423-443. doi:10.1007/s10404-010-0580-9.

Sundström, C., Nilsson, K., 1976. International Journal of Cancer 17, 565-577. doi:10.1002/ijc.2910170504.

Valero, A., Merino, F., Wolbers, F., Luttge, R., Vermes, I., Andersson, H., van den Berg, A., 2005. Lab Chip 5, 49-55. doi:10.1039/B415813J.

Wang, X., Becker, F., Gascoyne, P., 2002. Biochimica et Biophysica Acta 1564, 412-420. doi:10.1016/s0005-2736(02)00495-9.

Xie, X., Cheng, Z., Xu, Y., Liu, R., Li, Q., Cheng, J., 2017. Anal. Methods 9, 1201-1212. doi:10.1039/C6AY03326A.

Zhang, J., Lou, X., Jin, L., Zhou, R., Liu, S., Xu, N., Liao, D.J., 2014 Oncoscience 1, 407-422. doi:10.18632/oncoscience.61.

Zhao, Y., Wang, K., Chen, D., Fan, B., Xu, Y., Ye, Y., Wang, J., Chen, J., Huang, C., 2018. Biosensors and Bioelectronics 111, 138-143. doi:10.1016/j.bios.2018.04.015. 